\title{
Enhanced depth resolution in optical scanning holography using a configurable pupil
}

\author{
Haiyan Ou, ${ }^{1,2}$ Ting-Chung Poon, ${ }^{3}$ Kenneth K. Y. Wong, ${ }^{1}$ and Edmund Y. Lam ${ }^{1, *}$ \\ ${ }^{1}$ Department of Electrical and Electronic Engineering, University of Hong Kong, Pokfulam, Hong Kong, China \\ ${ }^{2}$ Institute of Applied Physics, University of Electronic Science and Technology of China, 610054, Chengdu, China \\ ${ }^{3}$ Bradley Department of Electrical and Computer Engineering, Virginia Tech, Blacksburg, Virginia 24061, USA \\ *Corresponding author: elam@eee.hku.hk
}

Received September 16, 2013; revised January 28, 2014; accepted January 29, 2014;

posted January 31, 2014 (Doc. ID 197685); published March 28, 2014

\begin{abstract}
The optical scanning holography (OSH) technique can capture all the three-dimensional volume information of an object in a hologram via a single raster scan. The digital hologram can then be processed to reconstruct individual sectional images of the object. In this paper, we present a scheme to reconstruct sectional images in OSH with enhanced depth resolution, where a spatial light modulator (SLM) is adopted as a configurable point pupil. By switching the SLM between two states, different Fresnel zone plates (FZPs) are generated based on the same optical system. With extra information provided by different FZPs, a depth resolution at $0.7 \mu \mathrm{m}$ can be achieved. (c) 2014 Chinese Laser Press
\end{abstract}

OCIS codes: $\quad$ (090.1995) Digital holography; (100.3190) Inverse problems; (100.3020) Image reconstruction-

restoration; (110.1758) Computational imaging.

http://dx.doi.org/10.1364/PRJ.2.000064

\section{INTRODUCTION}

Digital holography is a technique that seeks to capture the three-dimensional (3D) volume information of an object and to record it on a two-dimensional (2D) hologram electronically $[\underline{1}, 2]$. As one of the digital holographic techniques, optical scanning holography (OSH) can record the 2D holographic information of the $3 \mathrm{D}$ object by a single raster scan [3]. The idea of OSH was generated by Poon et al. when he was dealing with bipolar point spread functions in incoherent image processing $[\underline{4}, \underline{5}]$. The theory of OSH was then developed, and the first experiment was carried out in 1990 [6]. Since then, OSH has undergone great development and has found various applications ranging from biological microscopy $[\underline{7,}, \underline{8}]$ to remote sensing $[\underline{9}, \underline{10}]$ to $3 \mathrm{D}$ cryptography [11], etc.

Unlike laser scanning $[\underline{12}, \underline{13}]$ or confocal microscopy techniques $[14,15]$, where multiple $2 \mathrm{D}$ scans are needed to capture all the $3 \mathrm{D}$ information, OSH can greatly save on data acquisition time. While it is so powerful in capturing the 3D information, there is also a need in OSH to view the individual $2 \mathrm{D}$ planes of the object from the acquired digital hologram, which is known as sectioning or sectional image reconstruction. In the sectioning process, the challenge lies in the suppressing of the defocus noise, which is the undesired residue signal from neighboring sections. The conventional method of achieving this involves computing the convolution of the conjugate impulse response at the desired section with the hologram, but this approach suffers from large defocus noise [3]. To reduce the defocus noise, many methods including the Wiener filter [16], Wigner distribution [17], and inverse imaging [ $[\underline{18}, \underline{19}]$ have been demonstrated. Further development related to inverse imaging includes the development of a blind edge detection technique to locate the sections [20], compressed sensing [21], and edge-preserving regularization for sharper sections [22,23]. The use of a random-phase pupil is also shown to achieve better sectioning resolution [24], which requires the averaging of several independent holograms of the same object. Another method using a dual-wavelength laser source has been developed, with the depth resolution improved up to $2.5 \mu \mathrm{m}[\underline{25}, 26]$. More recently, Ou et al. have proposed a double-detection method, which has led to a depth resolution of $1 \mu \mathrm{m}$ by capturing holographic information of the same object at two different depth locations [27].

In this paper, we propose a sectioning method based on a configurable point pupil for achieving enhanced depth resolution. A spatial light modulator (SLM) is used as the switchable pupil, which generates different Fresnel zone plates (FZPs) for the same OSH system. We can then make use of the extra information provided by the different FZPs to achieve a better depth resolution. The proposed method is easier to realize in practice compared to some of the methods mentioned above.

\section{PRINCIPLE}

The OSH system setup is shown in Fig. 1 . In the optical system, the laser source centered at $\omega$ is divided into two parts by a beam splitter (BS1), of which one passes through a pupil function $p_{1}(x, y)$ to have a spherical wavefront on the object. Meanwhile, the other path first has a frequency shift $\Omega$ via an acousto-optic frequency shifter (AOFS) and then arrives at a point pupil $p_{2}(x, y)$ to result in a planar wavefront on the object. The two coherent beams of different frequencies $\omega$ and $\omega+\Omega$ are then combined by a second beamsplitter (BS2) and are used to scan the object located at a distance $z$ away from the scanning mirror. Lens 3 is used to collect the light from the object. A photodetector collects the transmitted and scattered light from the object and converts it into an electronic signal. 


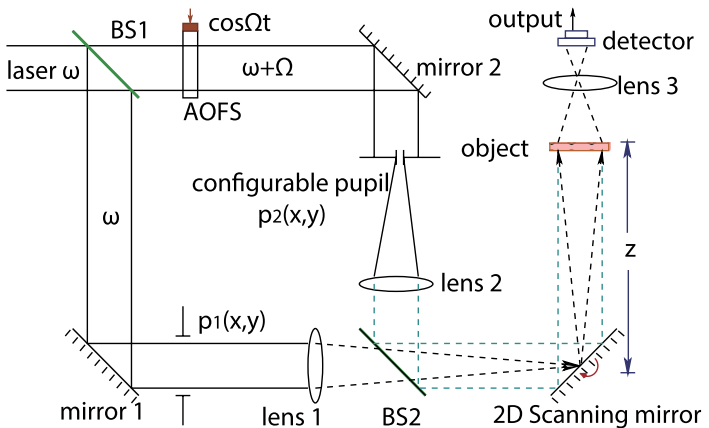

Fig. 1. Schematic of OSH

To recover the complex scattered wavefront from the object, an electronic demodulator and a low-pass filter are used to process the measured holograms, the details of which can be found in [28].

The optical transfer function (OTF) of the OSH system can be expressed as [28]

$$
\begin{aligned}
\mathcal{H}\left(k_{x}, k_{y} ; z\right)= & \exp \left[j \frac{z}{2 k_{0}}\left(k_{x}^{2}+k_{y}^{2}\right)\right] \\
& \times \iint_{-\infty}^{\infty} p_{1}^{*}\left(x^{\prime}, y^{\prime}\right) p_{2}\left(x^{\prime}+\frac{f}{k_{0}} k_{x}, y^{\prime}+\frac{f}{k_{0}} k_{y}\right) \\
& \times \exp \left[j \frac{z}{f}\left(x^{\prime} k_{x}+y^{\prime} k_{y}\right)\right] \mathrm{d} x^{\prime} \mathrm{d} y^{\prime},
\end{aligned}
$$

where $x, y, z$ are the spatial coordinates, $k_{x}$ and $k_{y}$ are the corresponding lateral spatial frequency coordinates, $f$ is the focal length of lens 1 and lens 2 , and $k_{0}=2 \pi / \lambda$ is the wavenumber of the optical source, with $\lambda$ representing the wavelength of the optical source.

The description and the imaging equation so far are similar to the conventional OSH setup. However, our scheme actually differs in two important aspects. First, we make use of an SLM to realize the pupil function $p_{2}(x, y)$, making it much more flexible and programmable. For our purpose here, we switch the SLM to two configurations, denoted as $p_{2 a}(x, y)$ and $p_{2 b}(x, y)$. In principle, it is also possible to design the SLM for more pupil functions, which may be combined with further computation for better sectioning capability. Second, the imaging process is also different, in that we take one measurement for each pupil configuration. Specifically, we first set $p_{2 a}=\delta(x, y)$ and record the first hologram $g_{1}(x, y)$; then, we tune the SLM to a second configuration and obtain the other hologram $g_{2}(x, y)$. The requirement for the second configuration is that $p_{2 b}(x, y)$ is sufficiently different from $p_{2 a}(x, y)$ to maximize the information content from the two measurements. In what follows, we will show that even with a simple shift of the impulse such that $p_{2 b}(x, y)=$ $\delta\left(x-x_{0}, y-y_{0}\right)$, where $x_{0}$ and $y_{0}$ are certain nonzero constants, we can increase the depth resolution of the OSH system significantly.

For the first hologram, the impulse response of the OSH system [with $p_{1}(x, y)=1$ and $p_{2 a}(x, y)=\delta(x, y)$ ] can be derived from Eq. (1) as

$$
h_{1}(x, y ; z)=j \frac{1}{\lambda z} \exp \left\{j \frac{\pi}{\lambda z}\left(x^{2}+y^{2}\right)\right\}
$$

where the impulse response $h(x, y ; z)$ is the Fourier transform of the OTF $\mathcal{H}\left(k_{x}, k_{y} ; z\right)$. Given an input object with an intensity distribution $\varphi(x, y, z)$, the resulting hologram is therefore

$$
g_{1}(x, y)=\sum_{i=1}^{n} \varphi\left(x, y ; z_{i}\right) * h_{1}\left(x, y ; z_{i}\right)
$$

where $*$ denotes the $2 \mathrm{D}$ convolution operation and $z_{i}$ represents the depth location of the $i$ th section, where we have assumed that we can discretize the object into $n$ sections.

For the second measurement, with $p_{1}(x, y)=1$ and $p_{2 b}(x, y)=\delta\left(x-x_{0}, y-y_{0}\right)$, the shifted OTF can be simplified as

$$
\begin{aligned}
\mathcal{H}_{2}\left(k_{x}, k_{y} ; z\right)= & \exp \left[j \frac{z}{2 k_{0}}\left(k_{x}^{2}+k_{y}^{2}\right)\right] \\
& \times \iint_{-\infty}^{\infty} \delta\left[\left(x^{\prime}-x_{0}\right)+\frac{f}{k_{0}} k_{x},\left(y^{\prime}-y_{0}\right)+\frac{f}{k_{0}} k_{y}\right] \\
& \times \exp \left[j \frac{z}{f}\left(x^{\prime} k_{x}+y^{\prime} k_{y}\right)\right] \mathrm{d} x^{\prime} \mathrm{d} y^{\prime},
\end{aligned}
$$

the impulse response thus can be expressed as

$$
h_{2}(x, y ; z)=j \frac{1}{\lambda z} \exp \left\{j \frac{\pi}{\lambda z}\left[\left(x-x_{0}\right)^{2}+\left(y-y_{0}\right)^{2}\right]\right\}
$$

and the corresponding hologram is

$$
g_{2}(x, y)=\sum_{i=1}^{n} \varphi\left(x, y ; z_{i}\right) * h_{2}\left(x, y ; z_{i}\right)
$$

We can combine the two hologram recordings into a single imaging equation as follows. Let $\mathbf{g}_{1}$ and $\mathbf{g}_{2}$ denote the lexicographical ordering of the holograms $g_{1}(x, y)$ and $g_{2}(x, y)$, respectively. In a similar way, the object at depth $i$ becomes $\varphi_{i}$. Its convolution with the impulse response at $z_{i}$ is denoted by the matrix-vector product $H_{1}\left(z_{i}\right) \varphi_{i}$ for the first hologram and $H_{2}\left(z_{i}\right) \varphi_{i}$ for the second one. The overall imaging is then represented by

$$
\begin{aligned}
\mathbf{g} & =\left[\begin{array}{l}
\mathbf{g}_{1} \\
\mathbf{g}_{2}
\end{array}\right] \\
& =\left[\begin{array}{llll}
\mathrm{H}_{1}\left(z_{1}\right) & \mathrm{H}_{1}\left(z_{2}\right) & \ldots & \mathrm{H}_{1}\left(z_{n}\right) \\
\mathrm{H}_{2}\left(z_{1}\right) & \mathrm{H}_{2}\left(z_{2}\right) & \ldots & \mathrm{H}_{2}\left(z_{n}\right)
\end{array}\right]\left[\begin{array}{c}
\boldsymbol{\varphi}_{1} \\
\boldsymbol{\varphi}_{2} \\
\vdots \\
\boldsymbol{\varphi}_{n}
\end{array}\right]+\left[\begin{array}{c}
\epsilon_{1} \\
\epsilon_{2} \\
\vdots \\
\epsilon_{n}
\end{array}\right] \\
& =\left[\begin{array}{c}
\mathrm{H}_{1} \\
\mathrm{H}_{2}
\end{array}\right] \boldsymbol{\varphi}+\epsilon \\
& =\mathrm{H} \varphi+\epsilon,
\end{aligned}
$$

where $\epsilon_{1}$ to $\epsilon_{n}$ represent the respective observation noise values. $\mathrm{H}_{1}$ and $\mathrm{H}_{2}$ denote the matrices of the first and second measurement, respectively, and $\mathrm{H}$ encapsulates all the impulse responses, while the object at various sections is represented by $\varphi$.

Our objective in sectioning is to recover the object vector $\varphi$ from the observation $\mathbf{g}$. This process can be viewed as a minimization problem [18]: 


$$
\boldsymbol{\varphi}_{\text {est }}=\arg \min _{\boldsymbol{\varphi}}\|\mathrm{H} \boldsymbol{\varphi}-\mathbf{g}\|_{2}^{2}+\lambda\|\mathrm{C} \boldsymbol{\varphi}\|_{2}^{2},
$$

where $\|\cdot\|_{2}$ denotes the $\ell_{2}$ norm, $\lambda>0$ is a penalty parameter, and $\mathrm{C}$ stands for the Laplacian operator. Other regularization methods, such as $\ell_{1}$ norm or total variation norm, have shown to have better results $[23,29,30]$; however, we choose $\ell_{2}$-norm regularization here to have a better comparison with both the inverse imaging method [19] and the double-detection technique [27].

The solution to this problem can be obtained as [ㅌ]

$$
\left(\mathrm{H}^{+} \mathrm{H}+\lambda \mathrm{C}^{+} \mathrm{C}\right) \varphi_{\text {est }}=\mathrm{H}^{+} \mathbf{g},
$$

where $\mathrm{H}^{+}$indicates the conjugate transpose of $\mathrm{H}$. We use the conjugate gradient method to solve this problem, the details of which are given in [31].

\section{SIMULATION AND ANALYSIS}

In this section, we analyze the sectioning results of the proposed method with an object having two and three dominant sections. We also make a comparison of the proposed method with double detection and study the effect of measurement error.

\section{A. Sectioning with Two Sections}

The proposed method is first demonstrated with an object that contains two sections at $z_{1}=34 \mathrm{~mm}$ and $z_{2}=34.0007 \mathrm{~mm}$, with the section distance equal to $0.7 \mu \mathrm{m}$. Each section has a size of $1 \mathrm{~mm} \times 1 \mathrm{~mm}$ in the lateral directions and is sampled to $512 \times 512$ pixels, as shown in Figs. 2 (a) and 2(b). The object

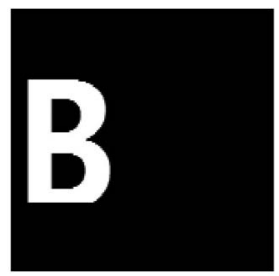

(a) section 1

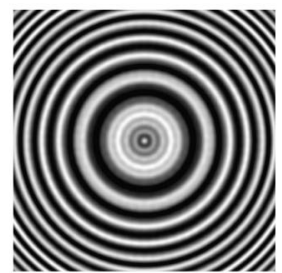

(c) real part of the FZP at $z_{1}$ with $p_{2 a}(x, y)$

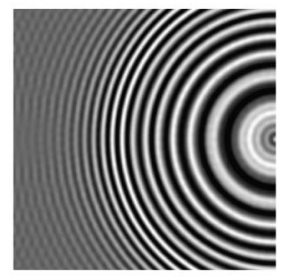

(e) real part of the FZP at $z_{1}$ with $p_{2 b}(x, y)$

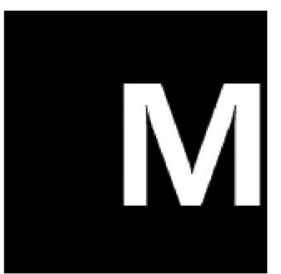

(b) section 2

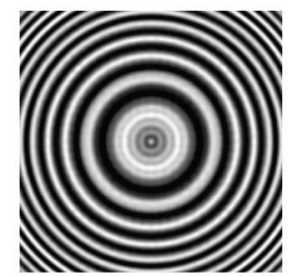

(d) real part of the FZP at $z_{2}$ with $p_{2 a}(x, y)$

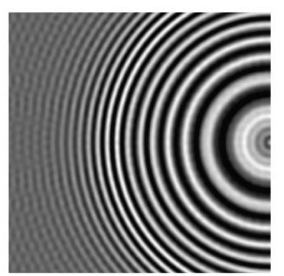

(f) real part of the FZP at $z_{2}$ with $p_{2 b}(x, y)$
Fig. 2. Two sections together with the real part of the FZPs for each scan. is illuminated by a He-Ne laser with the wavelength centered at $632 \mathrm{~nm}$. The configurable pupil $p_{2}(x, y)$ is realized by a liquid-crystal-on-silicon (LCoS) transmissive SLM with an $8 \mu \mathrm{m}$ pixel size, which can switch between two configurations: (1) $p_{2 a}(x, y)=\delta(x, y)$ and (2) $p_{2 b}(x, y)=\delta\left(x-x_{0}, y-y_{0}\right)$, with $x_{0}=0.48 \mathrm{~mm}$ and $y_{0}=0 \mathrm{~mm}$.

In the simulation, we use an OSH simulator to evaluate the performance of the proposed method, in which the finite diameter of the apertures and different kinds of noise are taken into consideration. The details of the OSH simulator can be found in [26]. Here, in the proposed method, the diameter of the collimated beam is $D=40 \mathrm{~mm}$ and the focal length is $f=50 \mathrm{~mm}$, which lead to a system numerical aperture [NA, where NA $=\sin (D / 2 f)]$ equal to 0.3894 . Both the thermal noise and the Poisson noise are evaluated in the simulator: the former is a white Gaussian noise with an energy per bandwidth at $\sigma_{0}^{2}\left(\sigma_{0}=12.75\right)$, while the other has a Gaussian distribution $\mathcal{N}\left(\bar{p}, c \bar{p}^{2}\right)$, where $\bar{p}$ denotes the mean value of the detected hologram signal amplitude and $c=0.0025$.

The measured FZPs with each configuration for the two sections are shown in Fig. 2. One can observe that as the pupil switches to $p_{2 b}(x, y)=\delta\left(x-x_{0}, y-y_{0}\right)$, the center of the FZP shifts $0.54 \mathrm{~mm}$ along the $x$ axis as well. As a result, the opaque and transparent zones are different in the observation area, which indicates that the spatial sampling rate of the FZPs will change as they shift away from the center. Accordingly, extra information can be captured during the second measurement, with which we can expect to allow for better sectioning. Convolving these FZPs with the object results in the holograms shown in Fig. 3.

These holograms become the input to the sectional image reconstruction described in the previous section. For comparison, we have implemented three different methods for sectioning: (1) the conventional method, (2) the conjugate gradient method with a single measurement, and (3) the proposed method with two measurements. The results are shown in Fig. 4. It can be observed that neither the conventional method nor the conjugate gradient method with a single measurement provides acceptable sectioning results to distinguish the different sections. On the other hand, with the proposed method, thanks to the extra information captured during the second measurement, each section is clearly separated with a

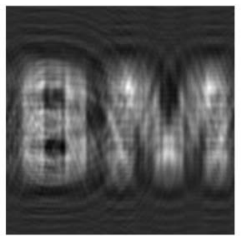

(a) real part of $g_{1}$

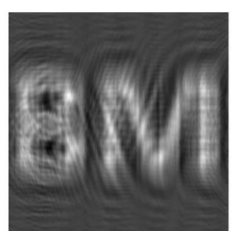

(c) real part of $g_{2}$

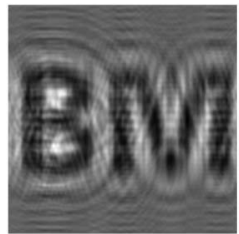

(b) imaginary part of $g_{1}$

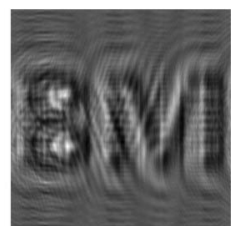

(d) imaginary part of $g_{2}$
Fig. 3. Holograms containing two-sectional images of the object with different pupil functions (a), (b) for $x_{0}=y_{0}=0$ and (c), (d) for $x_{0}=0.48 \mathrm{~mm}, y_{0}=0$. 


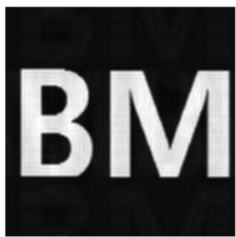

(a) $z_{1}$

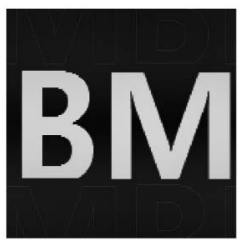

(c) $z_{1}$

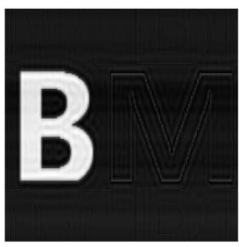

(e) $z_{1}$

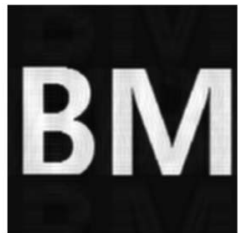

(b) $z_{2}$

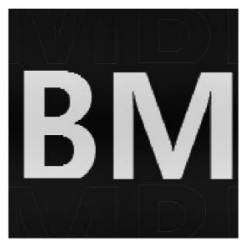

(d) $z_{2}$

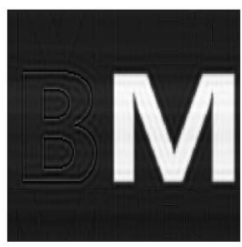

(f) $z_{2}$
Fig. 4. Sectioning results using (a), (b) the conventional method; (c), (d) the conjugate-gradient-based method with a single measurement; (e), (f) the conjugate-gradient-based method with the proposed method.

small amount of defocus noise. This indicates that the depth resolution can reach $0.7 \mu \mathrm{m}$ in this case. The measured signalto-noise rate (SNR) is $38.5 \mathrm{~dB}$ in the simulation.

The extra information captured during the second measurement is highly related to the correlation between the matrices $\mathrm{H}_{1}$ and $\mathrm{H}_{2}$ according to Eq. (7). The correlation of $\mathrm{H}_{1}$ and $\mathrm{H}_{2}$ can be evaluated using the following equation [32]:

$\mathcal{N}=\sum_{x=1}^{N} \sum_{y=1}^{M} \frac{\sum_{i=1}^{N} \sum_{j=1}^{M} \mathrm{H}_{1}(x+i, y+j) \cdot \mathrm{H}_{2}(i, j)}{\sqrt{\sum_{i=1}^{N} \sum_{j=1}^{M} \mathrm{H}_{1}(x+i, y+j)^{2}} \cdot \sqrt{\sum_{i=1}^{N} \sum_{j=1}^{M} \mathrm{H}_{2}(i, j)^{2}}}$.

As the difference between $\mathrm{H}_{1}$ and $\mathrm{H}_{2}$ is mainly caused by the lateral shift $x_{0}$, it is therefore useful to study the relationship between $x_{0}$ and the correlation of $\mathrm{H}_{1}$ and $\mathrm{H}_{2}$, as well as the relationship between $x_{0}$ and the system resolution. The results are shown in Fig. 5. There are two important features one can observe from this plot: (1) the correlation between the two matrices $\mathrm{H}_{1}$ and $\mathrm{H}_{2}$ decreases as $x_{0}$ increases, which is in accordance with our expectation, as a larger $x_{0}$ would lead to less similarity between $\mathrm{H}_{1}$ and $\mathrm{H}_{2}$; (2) as $x_{0}$ increases, the depth resolution would improve from 7.5 to $0.7 \mu \mathrm{m}$. This is because as the center of the FZP shifts away, the transition between the opaque and transparent zones becomes faster, which indicates that the spatial sampling rate of the FZPs becomes higher. This would provide us with more extra information for the hologram, which results in an enhanced depth resolution.

\section{B. Comparison with the Double-Detection Method}

From the analysis in the previous section, we can see that there is a trade-off between the lateral shift $x_{0}$ and the depth

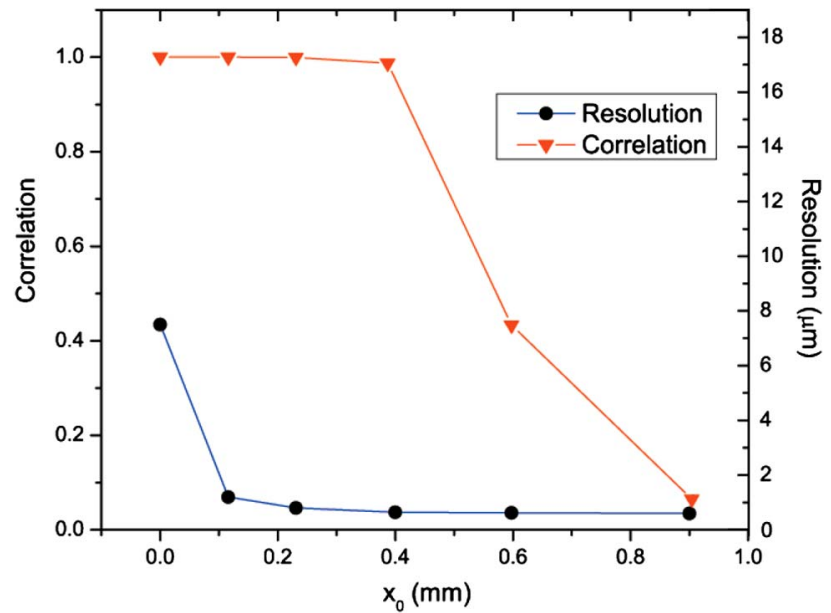

Fig. 5. Relationship between $x_{0}$ and the resolution and the relationship between $x_{0}$ and the correlation of $\mathrm{H}_{1}$ and $\mathrm{H}_{2}$.

resolution. This is also the case for the double-detection method, where the trade-off exists between the shift in depth ( $z$ axis) and the sectioning resolution [27]. In order to evaluate the performance of the proposed method and the doubledetection method, it is necessary to make a comparison between the two.

We first compare the relationship between the spatial shift and the correlation of $\mathrm{H}_{1}$ and $\mathrm{H}_{2}$, as is shown in Fig. 6 . It can be seen that with the same amount of shift, the lateral displacement with the proposed method would introduce more difference (i.e., less similarity and smaller correlation) between the two matrices representing the two OTFs compared to the double-detection method with displacement along the $z$ axis.

We have also compared the relationship between the spatial shift and the resolution. The result is shown in Fig. 7 . One can observe that the two curves hold the same trend, except that the one with the proposed method changes much faster, which is in accordance with the analysis of the correlation above. To achieve a depth resolution up to $1 \mu \mathrm{m}$, one needs to move the object along the $z$ axis for about 15 to $20 \mathrm{~mm}$ by using the double-detection technique, while with the proposed method, the lateral shift of the pupil $p_{2}$ is only around $0.1 \mathrm{~mm}$.

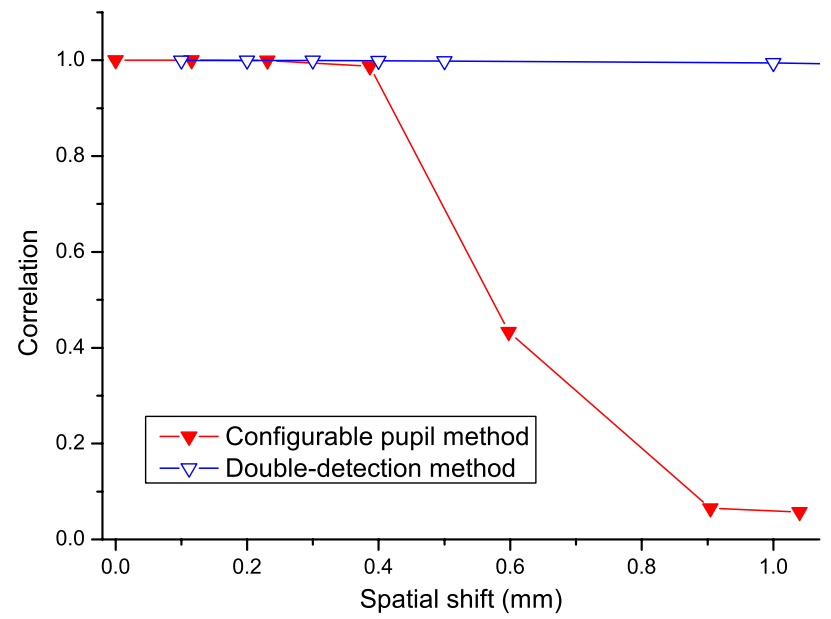

Fig. 6. Relationship between spatial shift and the correlation of $\mathrm{H}_{1}$ and $\mathrm{H}_{2}$. 


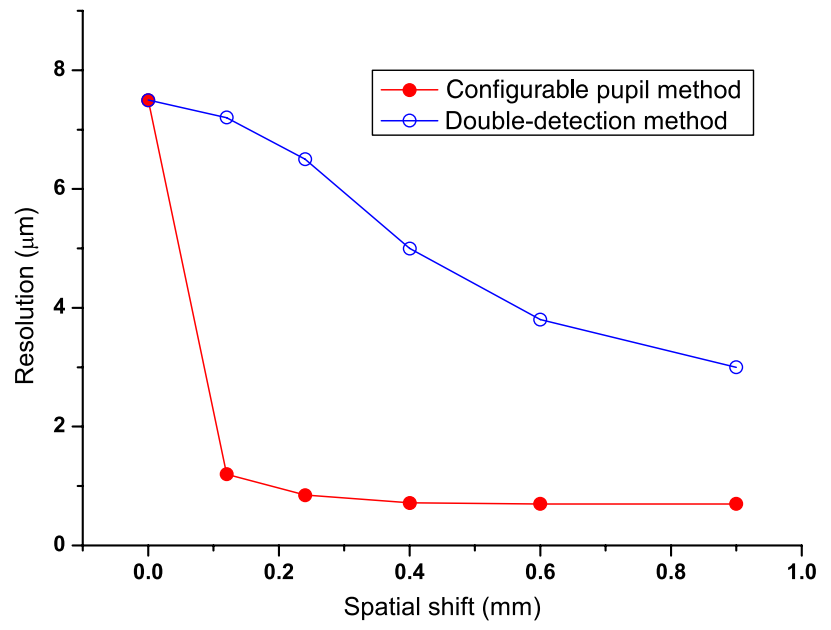

Fig. 7. Relationship between spatial shift and resolution.

It is worth mentioning that due to the SLM used in the system, there is no need to move the pupil $p_{2}$ itself in the actual optical system; one only needs to adjust the power distribution applied on the SLM along the $x$ axis for several pixels, which is more time efficient.

\section{Sectioning with Three Sections}

We then evaluate the performance of the proposed method with an object with three sections, as is shown in Fig. 8 . The location of the three sections are $z_{1}=34 \mathrm{~mm}, z_{2}=$ $34.0007 \mathrm{~mm}$, and $z_{3}=34.0014 \mathrm{~mm}$, respectively. The $\mathrm{OSH}$ simulator is also used to perform the simulation, in which the system NA and the noise are kept the same as before. The pupil function also switches between two states: (1) $p_{2 a}(x, y)=\delta(x, y)$ and (2) $p_{2 b}(x, y)=\delta\left(x-x_{0}, y-y_{0}\right)$, with $x_{0}=0.48 \mathrm{~mm}$ and $y_{0}=0 \mathrm{~mm}$. The holograms from each scan of the object are shown in Fig. 9 .

The sectioning results for this three-section object are shown in Fig. 10, in which the sectioning effect of the traditional method, the conjugate gradient method with a single measurement, and our proposed method with two measurements are compared. The proposed method also outperforms the other two in suppressing the defocus noise, which indicates that it can handle objects of multiple sections as well.

\section{Measurement Error Analysis}

Because we need to switch the SLM between two states, there would be inevitable measurement error in practice. However, unlike the double-detection method in [27], where the object itself is displaced along the $z$ axis, with configurable pupils there is no need to move any part of the optical setup, which should give rise to a smaller amount of the measurement

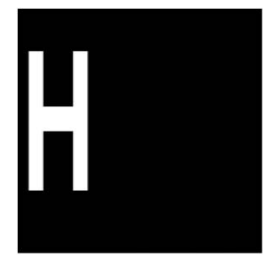

(a) section 1

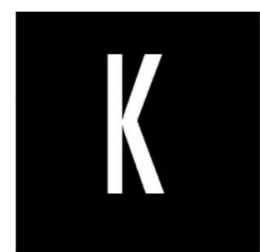

(b) section 2 (g) $z_{1}$

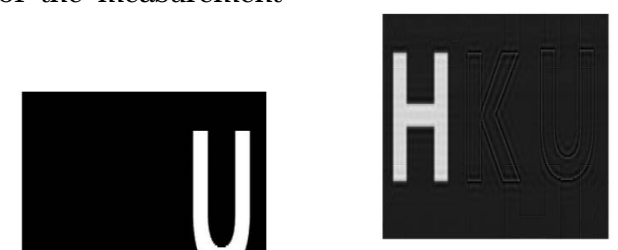

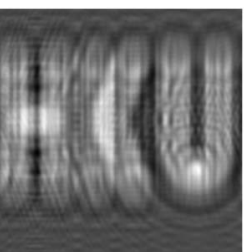

(a) real part of $g_{1}$

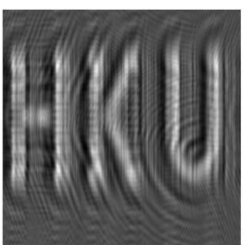

(c) real part of $g_{2}$

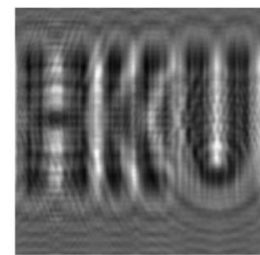

(b) imaginary part of $g_{1}$

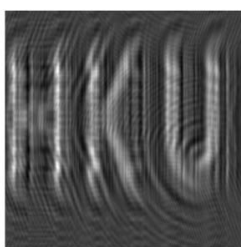

(d) imaginary part of $g_{2}$

Fig. 9. Holograms containing three-section images of the object with different pupil functions (a), (b) for $x_{0}=y_{0}=0$ and (c), (d) for $x_{0}=0.48 \mathrm{~mm}, y_{0}=0$.

error. The error mostly comes from the manufacturing defect of the pixel size of the SLM.

We use the same two-section object to test the effect of measurement error, with $x_{0}=0.48 \mathrm{~mm}$ for the second measurement. Here we define $\Delta x$ as the measurement error along the $x$ axis, so the pupil function $p_{2 b}(x, y)$ actually becomes $p_{2 b}(x, y)=\delta\left(x-x_{0}^{\prime}, y-y_{0}\right)$, with $x_{0}^{\prime}=x_{0}+\Delta x$ and $y_{0}=0 \mathrm{~mm}$. Three different situations are considered in the simulation: (1) $\Delta x=0 \mathrm{~mm}, z_{1}=34 \mathrm{~mm}, z_{2}=34.0007 \mathrm{~mm}$; (2) $\Delta x=100 \mathrm{~nm}, z_{1}=34 \mathrm{~mm}, z_{2}=34.0007 \mathrm{~mm}$; (3) $\Delta x=$ $100 \mathrm{~nm}, \quad z_{1}=34 \mathrm{~mm}, \quad z_{2}=34.01 \mathrm{~mm}$. The corresponding sectioning results using the proposed method are shown in Fig. 11. One can see that it is hard to distinguish the two sections when the measurement error reaches $100 \mathrm{~nm}$. However, as the section distance increases up to $10 \mu \mathrm{m}$, this

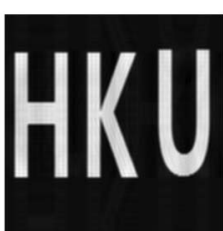

(a) $z_{1}$

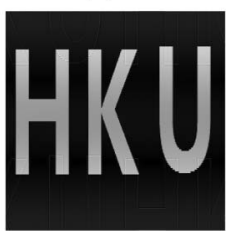

(d) $z_{1}$ Fig. 10. Sectioning results using (a)-(c) the conventional method;
(d)-(f) the conjugate-gradient-based method with a single measurement; (g)-(i) the conjugate-gradient-based method with the proposed method. 


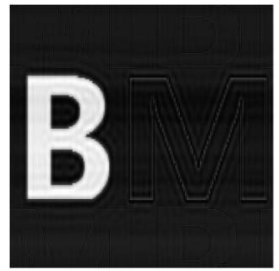

(a) $z_{1}$

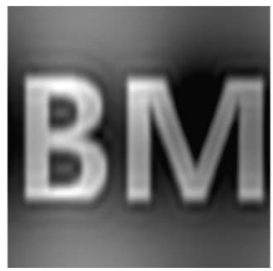

(c) $z_{1}$

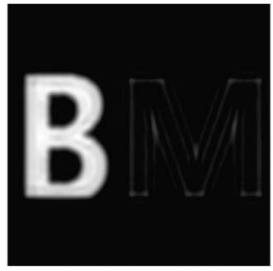

(e) $z_{1}$

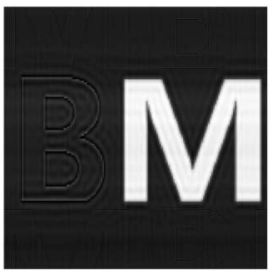

(b) $z_{2}$

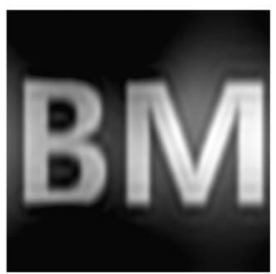

(d) $z_{2}$

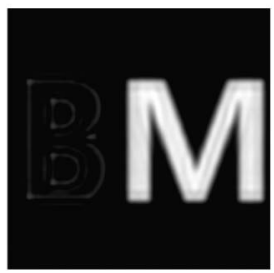

(f) $z_{2}$
Fig. 11. Sectioning results using the proposed method with (a)-(b) $\Delta x=0 \mathrm{~nm}, z_{1}=34 \mathrm{~mm}, z_{2}=34.0007 \mathrm{~mm}$; (c)-(d) $\Delta x=100 \mathrm{~nm}$, $z_{1}=34 \mathrm{~mm}, z_{2}=34.0007 \mathrm{~mm}$; (e)-(f) $\Delta x=100 \mathrm{~nm}, z_{1}=34 \mathrm{~mm}$, $z_{2}=34.01 \mathrm{~mm}$.

measurement error would be tolerated, leaving only a small amount of defocus noise. This indicates that the depth resolution has been degraded from 0.7 to around $10 \mu \mathrm{m}$ when measurement error $\Delta x=100 \mathrm{~nm}$.

We have also measured the relationship between the measurement error of the proposed method and the system depth resolution, which is shown in Fig. 12. As one would expect, the depth resolution would degrade as the measurement error increases. The depth resolution would degrade to $10 \mu \mathrm{m}$ when the measurement error reaches $100 \mathrm{~nm}$. For comparison, we also show the relationship between the measurement error and the system depth resolution of the double-detection

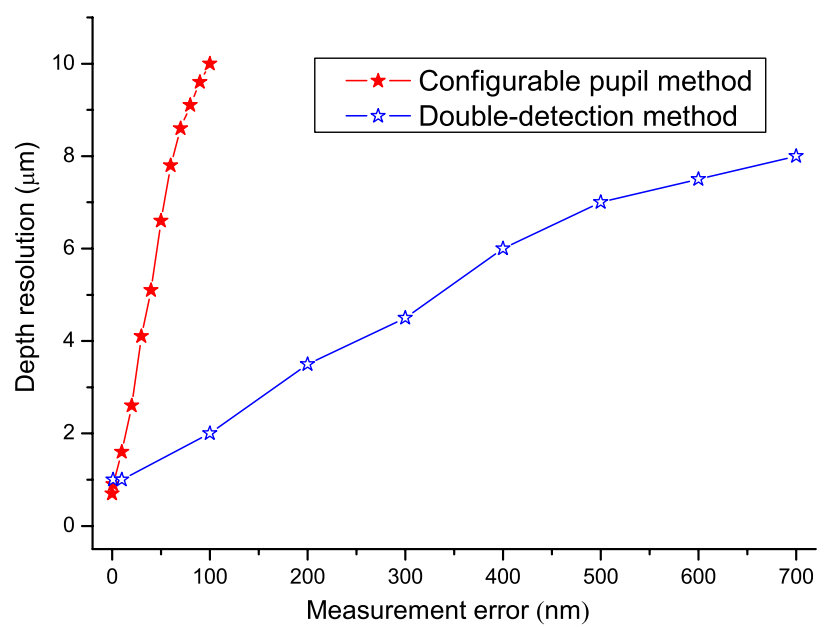

Fig. 12. Relationship between measurement error and the depth resolution. method here. One can see that the proposed method with a configurable pupil is more sensitive to measurement error compared to the double-detection method. This is due to the fact that the matrix $\mathrm{H}$ changes more quickly with the lateral spatial shift than the $z$ axis spatial shift, as discussed before.

\section{CONCLUSIONS}

A method has been proposed to reconstruct a sectional image using a configurable point pupil, which can be realized with a commercially available LCoS SLM. By controlling the voltage applied on each pixel of the SLM, the point pupil can be switched between two configurations. This can create two different sets of FZPs, with which extra information about the object can be extracted. By combining the holograms recorded during each scan process, we have shown that better sectioning results are possible.

\section{ACKNOWLEDGMENTS}

This research was supported in part by the Research Grants Council of the Hong Kong Special Administrative Region, China, under Projects HKU 7138/11E and 7131/12E. H. Ou is also grateful to the National Science Foundation of China (Grant 61107018) and the Fundamental Research Funds for the Central Universities (ZYGX2011J033), which allow her to enter into this collaborative research.

\section{REFERENCES}

1. J. W. Goodman and R. W. Lawrence, "Digital image formation from electronically detected holograms," Appl. Phys. Lett. 11, 77-79 (1967)

2. J. W. Goodman, "Digital image formation from holograms: early motivations and modern capabilities," in OSA Topical Meeting in Digital Holography and Three-Dimensional Imaging (2007), paper JMA1.

3. T.-C. Poon, Optical Scanning Holography with MATLAB (Springer, 2007).

4. T.-C. Poon and A. Korpel, "Optical transfer function of an acousto-optic heterodyning image processor," Opt. Lett. 4, 317-319 (1979).

5. T.-C. Poon, "Scanning holography and two-dimensional image processing by acousto-optic two-pupil synthesis," J. Opt. Soc. Am. A 2, 521-527 (1985).

6. T.-C. Poon, B. D. Duncan, M. H. Wu, K. Shinoda, and Y. Suzuki, "Real-time optical holography using a spatial light modulator," Jpn. J. Appl. Phys. 29, L1840-L1842 (1990).

7. J. Swoger, M. Martínez-Corral, J. Huisken, and E. H. K. Stelzer, "Optical scanning holography as a technique for high-resolution three-dimensional biological microscopy,” J. Opt. Soc. Am. A 19 1910-1918 (2002).

8. G. Indebetouw and W. Zhong, "Scanning holographic microscopy of three-dimensional fluorescent specimens," J. Opt. Soc. Am. A 23, 1699-1707 (2006).

9. B. W. Schilling and G. C. Templeton, "Three-dimensional remote sensing by optical scanning holography," Appl. Opt. 40, 5474-5481 (2001).

10. T. Kim, T.-C. Poon, and G. Indebetouw, "Depth detection and image recovery in remote sensing by optical scanning holography," Opt. Eng. 41, 1331-1338 (2002).

11. T.-C. Poon, T. Kim, and K. Doh, "Optical scanning cryptography for secure wireless transmission," Appl. Opt. 42, 6496-6503 (2003).

12. W. Denk, J. Strickler, and W. Webb, "Two-photon laser scanning fluorescence microscopy," Nature 248, 73-76 (1990).

13. J. S. Ploem, "Laser scanning fluorescence microscopy," Appl. Opt. 26, 3226-3231 (1987). 
14. S. Nie, D. Chiu, and R. Zare, "Probing individual molecules with confocal fluorescence microscopy," Science 266, 1018-1021 (1994).

15. J. Korlach, P. Schwille, W. Webb, and G. Feigenson, "Characterization of lipid bilayer phases by confocal microscopy and fluorescence correlation spectroscopy," Proc. Natl. Acad. Sci. USA 96, 8461-8466 (1999).

16. T. Kim, "Optical sectioning by optical scanning holography and a Wiener filter," Appl. Opt. 45, 872-879 (2006).

17. H. Kim, S.-W. Min, B. Lee, and T.-C. Poon, "Optical sectioning for optical scanning holography using phase-space filtering with Wigner distribution functions," Appl. Opt. 47, D164-D175 (2008).

18. E. Y. Lam, X. Zhang, H. Vo, T.-C. Poon, and G. Indebetouw, "Three-dimensional microscopy and sectional image reconstruction using optical scanning holography," Appl. Opt. 48, H113-H119 (2009).

19. X. Zhang, E. Y. Lam, and T.-C. Poon, "Reconstruction of sectional images in holography using inverse imaging," Opt. Express 16, 17215-17226 (2008).

20. X. Zhang, E. Y. Lam, T. Kim, Y. S. Kim, and T.-C. Poon, "Blind sectional image reconstruction for optical scanning holography," Opt. Lett. 34, 3098-3100 (2009).

21. X. Zhang and E. Y. Lam, "Sectional image reconstruction in optical scanning holography using compressed sensing," in IEEE International Conference on Image Processing (2010), pp. 3349-3352.

22. X. Zhang and E. Y. Lam, "Edge detection of three-dimensional object by manipulating pupil functions in optical scanning holography system," in IEEE International Conference on Image Processing (2010), pp. 3661-3664.
23. X. Zhang and E. Y. Lam, "Edge-preserving sectional image reconstruction in optical scanning holography," J. Opt. Soc. Am. A 27, 1630-1637 (2010).

24. Z. Xin, K. Dobson, Y. Shinoda, and T.-C. Poon, "Sectional image reconstruction in optical scanning holography using a randomphase pupil," Opt. Lett. 35, 2934-2936 (2010).

25. J. Ke and E. Y. Lam, "Using a dual-wavelength source for depth resolution enhancement in optical scanning holography," in OSA Topical Meeting in Digital Holography and ThreeDimensional Imaging (2011), paper DTuC30.

26. J. Ke, T.-C. Poon, and E. Y. Lam, "Depth resolution enhancement in optical scanning holography with a dual-wavelength laser source," Appl. Opt. 50, H285-H296 (2011).

27. H. Ou, T.-C. Poon, K. K. Wong, and E. Y. Lam, "Depth resolution enhancement in double-detection optical scanning holography," Appl. Opt. 52, 3079-3087 (2013).

28. T.-C. Poon, "On the fundamentals of optical scanning holography," Am. J. Phys. 76, 738-745 (2008).

29. H. Di, K. Zheng, X. Zhang, E. Y. Lam, T. Kim, Y. S. Kim, T.-C Poon, and C. Zhou, "Multiple-image encryption by compressive holography," Appl. Opt. 51, 1000-1009 (2012).

30. D. J. Brady, K. Choi, D. L. Marks, R. Horisaki, and S. Lim "Compressive holography," Opt. Express 17, 13040-13049 (2009)

31. Z.-Z. Bai and Z.-Q. Wang, "Restrictive preconditioners for conjugate gradient methods for symmetric positive definite linear systems," J. Comput. Appl. Math. 187, 202-226 (2006).

32. S. Wei and S. Lai, "Fast template matching based on normalized cross correlation with adaptive multilevel winner update," IEEE Trans. Image Process. 17, 2227-2235 (2008). 